\title{
Amenability, critical exponents of subgroups and growth of closed geodesics
}

\author{
Rhiannon Dougall ${ }^{1} \cdot$ Richard Sharp $^{1}$
}

Received: 30 April 2015 / Revised: 25 November 2015 / Published online: 11 December 2015 (C) The Author(s) 2015. This article is published with open access at Springerlink.com

\begin{abstract}
Let $\Gamma$ be a (non-elementary) convex co-compact group of isometries of a pinched Hadamard manifold $X$. We show that a normal subgroup $\Gamma_{0}$ has critical exponent equal to the critical exponent of $\Gamma$ if and only if $\Gamma / \Gamma_{0}$ is amenable. We prove a similar result for the exponential growth rate of closed geodesics on $X / \Gamma$. These statements are analogues of classical results of Kesten for random walks on groups and Brooks for the spectrum of the Laplacian on covers of Riemannian manifolds.
\end{abstract}

\section{Introduction}

Let $X$ be a connected simply connected and geodesically complete Riemannian manifold with sectional curvatures bounded between two negative constants. We call such an $X$ a pinched Hadamard manifold. Let $\Gamma$ be a non-elementary and convex co-compact group of isometries of $X$ (see Sect. 2 for precise definitions) and let $\Gamma_{0} \triangleleft \Gamma$ be a normal subgroup. We write $G=\Gamma / \Gamma_{0}$. We define the critical exponent of $\Gamma$ to be the abscissa of convergence of the Dirichlet series

$$
\sum_{g \in \Gamma} e^{-s d_{X}(o, g o)}
$$

for any choice of base point $o \in X$, and denote it by $\delta(\Gamma)$. The critical exponent $\delta\left(\Gamma_{0}\right)$ of $\Gamma_{0}$ is defined in the same way. The fact that $\Gamma$ is non-elementary and convex

\footnotetext{
Richard Sharp

R.J.Sharp@warwick.ac.uk

Rhiannon Dougall

R.Dougall@warwick.ac.uk

1 Mathematics Institute, University of Warwick, Coventry CV4 7AL, UK
} 
co-compact means that $\delta(\Gamma)>0$ and it is clear that $\delta\left(\Gamma_{0}\right) \leq \delta(\Gamma)$. It is natural to ask when we have equality and our main result will give a precise answer to this question, which will depend only on $G$ as an abstract group. (We will discuss the history of this and related problems in the next section.)

Before stating our result, we introduce an alternative formulation. Consider the quotient manifolds $M=X / \Gamma$ and $\widetilde{M}=X / \Gamma_{0} ; \widetilde{M}$ is a regular cover of $M$ with covering group $G$. Then $M$ has a countably infinite set $\mathcal{C}(M)$ of closed geodesics (which are not assumed to be prime). For $\gamma \in \mathcal{C}(M)$, we write $l(\gamma)$ for its length. For each $T>0$, the set $\{\gamma \in \mathcal{C}(M): l(\gamma) \leq T\}$ is finite and we can define a number $h=h(M)>0$ by

$$
h(M)=\lim _{T \rightarrow \infty} \frac{1}{T} \log \#\{\gamma \in \mathcal{C}(M): l(\gamma) \leq T\}
$$

(The limit exists and, in fact, $\lim _{T \rightarrow \infty} T e^{-h(M) T} \#\{\gamma \in \mathcal{C}(M): l(\gamma) \leq T\}=1 / h(M)$ [20,22].) Similarly, we write $\mathcal{C}(\widetilde{M})$ for the set of closed geodesics on $\widetilde{M}$. If $G$ is infinite then, for a given $T$, the set $\{\gamma \in \mathcal{C}(\widetilde{M}): l(\gamma) \leq T\}$ is infinite (since a single closed geodesic has infinitely many images under the action of $G$ ). However, we can obtain a finite quantity by choosing any relatively compact open subset $W$ of the unit-tangent bundle $S \widetilde{M}$ that intersects the non-wandering set for the geodesic flow and considering the set

$$
\mathcal{C}(\tilde{M}, W)=\{\gamma \in \mathcal{C}(\widetilde{M}): \hat{\gamma} \cap W \neq \varnothing\}
$$

where $\hat{\gamma}$ is the periodic orbit for the geodesic flow lying over the closed geodesic $\gamma$. Following [21], we then define $h(\widetilde{M}) \leq h(M)$ by

$$
h(\widetilde{M})=\limsup _{T \rightarrow \infty} \frac{1}{T} \log \#\{\gamma \in \mathcal{C}(\tilde{M}, W): l(\gamma) \leq T\}
$$

and this is independent of the choice of $W$. Again we may ask when we have equality.

It is well known that $h(M)=\delta(\Gamma)$, and both are equal to the topological entropy of the geodesic flow over $X / \Gamma$. Furthermore, the work of Paulin et al. in [21] shows that $h(\widetilde{M})=\delta\left(\Gamma_{0}\right)$ (and that the limsups in the definitions are, in fact, limits). We will discuss this further in Sect. 2.

Our main result is the following.

Theorem 1.1 Let $\Gamma$ be a convex co-compact group of isometries of a pinched Hadamard manifold $X$ and let $\Gamma_{0}$ be a normal subgroup of $\Gamma$. Then the following are equivalent:

(i) $\delta\left(\Gamma_{0}\right)=\delta(\Gamma)$,

(ii) $h\left(X / \Gamma_{0}\right)=h(X / \Gamma)$,

(iii) $G=\Gamma / \Gamma_{0}$ is amenable.

In view of the equivalence of (i) and (ii) discussed above, this will follow from Theorem 7.5 below, in which we prove the equivalence of (ii) and (iii). 
Remark 1.2 In fact, that (iii) implies (i) is a theorem of Roblin [26] (see also the expository account in [27]), which actually applies in the more general situation where $X$ is a CAT $(-1)$ space.

Remark 1.3 In the special case where $X=\mathbb{H}^{d+1}$ and $\delta(\Gamma)>d / 2$, the statement $\delta\left(\Gamma_{0}\right)=\delta(\Gamma)$ if and only if $G$ is amenable is a result of Brooks [6] (and holds when $\Gamma$ is geometrically finite, which is a more general condition than convex cocompactness). We discuss this in more detail in the next section. If $X=\mathbb{H}^{d+1}$ and $\Gamma$ is essentially free then Stadlbauer showed the same result holds without the assumption $\delta(\Gamma)>d / 2$ [30]. The class of essentially free groups includes all non-co-compact geometrically finite Fuchsian groups (i.e. discrete groups of isometries of $\mathbb{H}^{2}$ ) and all Schottky groups.

We conclude the introduction by outlining the contents of the paper. In the next section, we define the key concepts associated to groups that are mentioned above and discuss some history of this and analogous problems. Our approach to Theorem 1.1 is via dynamics. More precisely, we consider the geodesic flow over $M$ and $\widetilde{M}$ and a class of symbolic dynamical systems that model them. These symbolic systems belong to a class called countable state Markov shifts: we introduce these in Sect. 3 and define a key quantity called the Gurevič pressure. We also mention recent results of Stadlbauer that will be key to our analysis. In Sects. 4, 5 and 6, we consider the geodesic flows over $M$ and $\widetilde{M}$ and discuss how they may be modeled by symbolic systems, particular using a skew product construction to record information about lifts to the cover. Finally, in Sect. 7, we link various zeta functions defined by the closed geodesics to the Gurevič pressure and hence, using Stadlbauer's result, prove that the equality of $h(M)$ and $h(\widetilde{M})$ is equivalent to amenability of the covering group.

We are grateful to the referee for their careful reading of the paper, which has resulted in numerous corrections and improvements.

\section{Background and history}

Let $X$ be a pinched Hadamard manifold, i.e. a connected simply connected complete Riemannian manifold such that its sectional curvatures lie in a interval $\left[-\kappa_{1},-\kappa_{2}\right]$, for some $\kappa_{1}>\kappa_{2}>0$. Associated to $X$ is a well defined topological space $\partial X$ called the Gromov boundary. This can be defined to be the set of equivalence classes of geodesic rays emanating from a fixed base point, where two rays are equivalent it their distance apart is bounded above. Let $\Gamma$ be a group of isometries acting freely and properly discontinuously on $X$. We say that $\Gamma$ is non-elementary if it is not a finite extension of a cyclic group. Fix $o \in X$. Then the orbit $\Gamma o=\{g o: g \in \Gamma\}$ accumulates only on $\partial X$ and we call the set of accumulation points $L_{\Gamma}$ the limit set of $\Gamma$; this is independent of the choice of $o$. Let $C(\Gamma)$ denote the intersection of $X$ with the convex hull (with respect to the metric on $X$ ) of $L_{\Gamma}$. We say that $\Gamma$ is convex co-compact if $C(\Gamma) / \Gamma$ is compact. If $\Gamma$ is convex co-compact then $M=X / \Gamma$ has a compact core: a manifold with boundary $M_{0}$ which contains $\mathcal{C}(M)$.

Now suppose that $\Gamma_{0} \triangleleft \Gamma$ and that $\widetilde{M}=X / \Gamma_{0}$. Then $\widetilde{M}$ is a regular $G=\Gamma / \Gamma_{0^{-}}$ cover of $M$. Let $\pi: \widetilde{M} \rightarrow M$ denote the projection. It is shown in [21] that if $W$ 
is a relatively compact open subset of the unit-tangent bundle $S \widetilde{M}$ intersecting the non-wandering set for the geodesic flow $\widetilde{\phi}_{t}: S \widetilde{M} \rightarrow S \widetilde{M}$ for any $c>0$,

$$
\delta\left(\Gamma_{0}\right)=\lim _{T \rightarrow \infty} \frac{1}{T} \log \#\{\gamma \in \mathcal{C}(\tilde{M}, W): T-c<l(\gamma) \leq T\} .
$$

(To see this, take $F=0$ in Theorem 1.1 of [21].)

Lemma 2.1 We have $h(M)=\delta\left(\Gamma_{0}\right)$.

Proof If $\delta\left(\Gamma_{0}\right)>0$ when we may replace the condition $l(\gamma) \leq T$ with $T-c<$ $l(\gamma) \leq T$ without affecting the exponentional growth rate and conclude the result. On the other hand, if $\delta\left(\Gamma_{0}\right)=0$ then the simple inequality $h(\widetilde{M}) \leq \delta\left(\Gamma_{0}\right)$ gives $h(\widetilde{M})=0$.

A (countable) group $G$ is amenable if $\ell^{\infty}(G, \mathbb{R})$ admits an invariant mean, i.e. that there exists a bounded linear functional $v: \ell^{\infty}(G, \mathbb{R}) \rightarrow \mathbb{R}$ such that, for all $f \in \ell^{\infty}(G, \mathbb{R})$,

(1) $\inf _{g \in G} f(g) \leq v(f) \leq \sup _{g \in G} f(g)$; and

(2) for all $g \in G, v\left(f_{g}\right)=v(f)$, where $f_{g}(h)=f\left(g^{-1} h\right)$.

The concept was introduced by von Neumann in 1929. One sees immediately from this definition that finite groups are amenable by taking

$$
v(f)=\frac{1}{|G|} \sum_{g \in G} f(g) .
$$

An alternative criterion for amenability was given by Følner [10]: $G$ is amenable if and only if, for every $\epsilon>0$ and every finite set $\left\{g_{1} \ldots, g_{n}\right\} \subset G$, there exists a finite set $F \subset G$ such that $\#\left(F \cap g_{i} F\right) \geq(1-\epsilon) \# F, i=1, \ldots, n$. Using this criterion, it is easy to see that abelian groups are amenable and that, more generally, groups with subexponential growth are amenable [12]. Furthermore, since amenability is closed under extensions, solvable groups are amenable. In particular, there are amenable groups with exponential growth (e.g. lamplighter groups). On the other hand, a group containing the free group on two generators is not amenable and non-elementary Gromov hyperbolic groups (a class which includes the convex co-compact groups above) are not amenable.

There are numerous results that connect growth and spectral properties of groups and manifolds to amenability. The prototype is the following theorem of Kesten from 1959. Let $G$ be a countable group and let $p: G \rightarrow \mathbb{R}^{+}$be a symmetric probability distribution (i.e. $\sum_{g \in G} p(g)=1$ and $p\left(g^{-1}\right)=p(g)$ for all $g \in G$ ) such that its support, $\operatorname{supp}(p)$, generates $G$. This defines a symmetric random walk on $G$ with transition probabilities $P\left(g, g^{\prime}\right)=p\left(g^{-1} g^{\prime}\right)$. If we define $\lambda(G, P)$ to be the $\ell^{2}(G)$ spectral radius of $P$ then we have

$$
\lambda(G, P)=\limsup _{n \rightarrow \infty} P^{n}(g, g)^{1 / n}=\lim _{n \rightarrow \infty} P^{2 n}(g, g)^{1 / 2 n},
$$

for any $g \in G$. It is clear that $\lambda(G, P) \leq 1$. 
Theorem 2.2 (Kesten [14]) We have $\lambda(G, P)=1$ if and only if $G$ is amenable.

Note that, while $\lambda(G, P)$ depends on the probability $p$, whether or not it takes the value 1 depends only on the group $G$.

Subsequently, results inspired by Kesten's Theorem were obtained in a variety of other situations. In the setting of group theory, the most notable result is Grigorchuk's co-growth criterion [11] (see also Cohen [8]) for finitely generated groups. Recall that a finitely generated group $G$ may be written as $F / N$, where $F$ is a free group of rank $k$ and $N$ is a normal subgroup. If $|\cdot|$ denotes the word length on $F$ with respect to a free generating set then $\lim _{n \rightarrow \infty}(\#\{x \in F:|x|=n\})^{1 / n}=2 k-1$.

Theorem 2.3 (Grigorchuk [11]) We have

$$
\limsup _{n \rightarrow \infty}(\#\{x \in N:|x|=n\})^{1 / n}=2 k-1
$$

if and only if $G$ is amenable.

Subsequently, various extensions of this to graphs and (non-backtracking) random walks were obtained by Woess [31], Northshield [17,18] and Ortner and Woess [19].

In the setting of Riemannian manifolds, an analogue is provided by the following spectral result of Brooks. Let $M$ be a complete Riemannian manifold which is of "finite topological type", i.e. that it is topologically the union of finitely many simplices, and let $\widetilde{M}$ be a regular covering of $M$ with covering group $G$. Let $\lambda_{0}(M)$ and $\lambda_{0}(\widetilde{M})$ denote the infimum of the spectrum of the Laplace-Beltrami operator on $M$ and $\widetilde{M}$, respectively; then $\lambda_{0}(\widetilde{M}) \geq \lambda_{0}(M)$. Brooks showed that amenability of $G$ implied equality and that, together with an additional condition, the converse holds. More precisely, he proved the following.

\section{Theorem 2.4 (Brooks [6])}

(i) If $G$ is amenable then $\lambda_{0}(\tilde{M})=\lambda_{0}(M)$.

(ii) Let $\phi$ be the lift of a $\lambda_{0}(M)$-harmonic function to $\widetilde{M}$ and let $F$ be a fundamental region for $M$ on $\widetilde{M}$. Suppose that there is a compact $K \subset F$ such that

$$
\inf _{S} \frac{\int_{S} \phi^{2} d \text { Area }}{\int_{\operatorname{int}(S)} \phi^{2} d \text { Vol }}>0,
$$

where the infimum is taken over co-dimension 1 submanifolds $S$ that divide $F \backslash K$ into an interior and an exterior. If $\lambda_{0}(\widetilde{M})=\lambda_{0}(M)$ then $G$ is amenable.

(See also Brooks [5] for the case when $M$ is compact and $G$ is its fundamental group and Burger [7] for a shorter proof.) The Cheeger-type condition in part (ii) holds if, for example, $M$ is a convex co-compact quotient of the $(d+1)$-dimensional real hyperbolic space $\mathbb{H}^{d+1}$ and $\lambda_{0}(M)<d^{2} / 4$.

The problem of critical exponents was also first considered in the case $X=\mathbb{H}^{d+1}$. In the early 1980s, Rees [25] showed that if $\Gamma / \Gamma_{0}$ is abelian then we have equality (and her dynamical methods generalize to variable curvature). Soon afterwards, Brooks 
obtained a more general result as a corollary of Theorem 2.4. This is due to the fact that, for $X=\mathbb{H}^{d+1}, \delta(\Gamma)$ and $\lambda_{0}(M)$ are related by the formula $\lambda_{0}(M)=\delta(\Gamma)(d-\delta(\Gamma))$, provided $\delta(\Gamma)>d / 2$, with the same holding for $\delta\left(\Gamma_{0}\right)$ and $\lambda_{0}(\widetilde{M})$. In particular, if $\delta(\Gamma)>d / 2$ then $\lambda_{0}(M)<d^{2} / 4$ and so Theorem 2.4 implies the statement that $\delta\left(\Gamma_{0}\right)=\delta(\Gamma)$ if and only if $G$ is amenable.

More recently, Stadlbauer [30] and Jaerisch [13] have considered the relation between amenability and certain growth rates that occur in the study of skew product extensions of dynamical systems. It will be clear below that we are greatly indebted to this work in our analysis.

\section{Countable state Markov shifts and Gurevič pressure}

In this section we will define countable state Markov shifts and discuss some of their properties. Basic definitions and results are taken from chapter 7 of [15]. In the rest of the paper, we shall be concerned with finite state shifts and skew product extensions of these by a countable group, so we shall often specialise to these two cases.

Let $S$ be a countable set, called the alphabet, and let $A$ be a matrix, called the transition matrix, indexed by $S \times S$ with entries zero or one. We then define the space

$$
\Sigma_{A}^{+}=\left\{x=\left(x_{n}\right)_{n=0}^{\infty} \in S^{\mathbb{Z}^{+}}: A\left(x_{n}, x_{n+1}\right)=1 \forall n \in \mathbb{Z}^{+}\right\}
$$

with the product topology induced by the discrete topology on $S$. This topology is compatible with the metric $d(x, y)=2^{-n(x, y)}$, where

$$
n(x, y)=\inf \left\{n: x_{n} \neq y_{n}\right\}
$$

with $n(x, y)=\infty$ if $x=y$. If $S$ is finite then $\Sigma_{A}^{+}$is compact. We say that $A$ is locally finite if all its row and column sums are finite. Then $\Sigma_{A}^{+}$is locally compact if and only if $A$ is locally finite. (The skew product extensions we consider have this latter property.)

We define the (one-sided) countable state topological Markov shift $\sigma: \Sigma_{A}^{+} \rightarrow \Sigma_{A}^{+}$ by $(\sigma x)_{n}=x_{n+1}$. This is a continuous map. We will say that $\sigma$ is topologically transitive if it has a dense orbit and topologically mixing if, given non-empty open sets $U, V \subset \Sigma_{A}^{+}$, there exists $N \geq 0$ such that $\sigma^{-n}(U) \cap V \neq \varnothing$ for all $n \geq N$. We say that the matrix $A$ is irreducible if, for each $(i, j) \in S \times S$, there exists $n=n(i, j) \geq 1$ such that $A^{n}(i, j)>0$. For $A$ irreducible, set $p \geq 1$ to be the greatest common divisor of periods of periodic orbits $\sigma: \Sigma_{A}^{+} \rightarrow \Sigma_{A}^{+}$; this $p$ is called the period of $A$. We say that $A$ is aperiodic if $p=1$ or, equivalently, if there exists $n \geq 1$ such that $A^{n}$ has all entries positive. Suppose that $A$ is locally finite. Then $\sigma: \Sigma_{A}^{+} \rightarrow \Sigma_{A}^{+}$is topologically transitive if and only if $A$ is irreducible and $\sigma: \Sigma_{A}^{+} \rightarrow \Sigma_{A}^{+}$is topologically mixing if and only if $A$ is aperiodic.

Suppose that $A$ is irreducible but not aperiodic and fix $i \in S$. Then we may partition $S$ into sets $S_{l}, l=0, \ldots, p-1$, defined by

$$
S_{l}=\left\{j: A^{n p+l}(i, j)>0 \text { for some } n \geq 1\right\} .
$$


(This partition is independent of the choice of $i$.) For each $l$, let $A_{l}$ denote the restriction of $A$ to $S_{l} \times S_{l}$; then $\sigma: \Sigma_{A_{l}}^{+} \rightarrow \Sigma_{A_{l+1}}^{+}(\bmod p)$ and $A_{l}^{p}$ is aperiodic.

We say that an $n$-tuple $w=\left(w_{0}, \ldots, w_{n-1}\right) \in S^{n}$ is an allowed word of length $n$ if $A\left(w_{j}, w_{j+1}\right)=1$ for $j=0, \ldots, n-2$. We will write $\mathcal{W}^{n}$ for the set of allowed words of length $n$. If $w \in \mathcal{W}^{n}$ then we define the associated cylinder set $[w]$ by

$$
[w]=\left\{x \in \Sigma_{A}^{+}: x_{j}=w_{j}, \quad j=0, \ldots, n-1\right\} .
$$

For a function $f: \Sigma_{A}^{+} \rightarrow \mathbb{R}$, set

$$
V_{n}(f)=\sup \left\{|f(x)-f(y)|: x_{j}=y_{j}, \quad j=0, \ldots, n-1\right\} .
$$

We say that $f$ is locally Hölder continuous if there exist $0<\theta<1$ and $C \geq 0$ such that, for all $n \geq 1, V_{n}(f) \leq C \theta^{n}$. (There is no requirement of $V_{0}(f)$ and a locally Hölder $f$ may be unbounded.) For $n \geq 1$, we write

$$
f^{n}:=f+f \circ \sigma+\cdots+f \circ \sigma^{n-1} .
$$

Definition 3.1 Suppose that $\sigma: \Sigma_{A}^{+} \rightarrow \Sigma_{A}^{+}$is topologically transitive and let $f$ : $\Sigma_{A}^{+} \rightarrow \mathbb{R}$ be a locally Hölder continuous function. Following Sarig [28], we define the Gurevic pressure, $P_{G}(\sigma, f)$, of $f$ to be

$$
P_{G}(\sigma, f)=\limsup _{n \rightarrow \infty} \frac{1}{n} \log \sum_{\substack{\sigma^{n} x=x \\ x_{0}=a}} e^{f^{n}(x)},
$$

where $a \in S$. (The definition is independent of the choice of $a$.)

Remark 3.2 In [28], Sarig gives this definition in the case where $\sigma: \Sigma_{A}^{+} \rightarrow \Sigma_{A}^{+}$is topologically mixing. However, the above decomposition of $\Sigma_{A}^{+}=\Sigma_{A_{0}}^{+} \cup \cdots \cup \Sigma_{A_{p-1}}^{+}$, with $\sigma^{p}$ topologically mixing on each component, together with the regularity of the function $f$, shows that the same definition may be made in the topologically transitive case.

We now specialise to the case where $S$ is finite. In this situation, we call $\sigma: \Sigma_{A}^{+} \rightarrow$ $\Sigma_{A}^{+}$a (one-sided) subshift of finite type. The above definitions and results hold. If $f: \Sigma_{A}^{+} \rightarrow \mathbb{R}$ is Hölder continuous then $f$ is locally Hölder. Provided $\sigma: \Sigma_{A}^{+} \rightarrow \Sigma_{A}^{+}$ is topologically transitive, the Gurevič pressure $P_{G}(\sigma, f)$ agrees with the standard pressure $P(\sigma, f)$, defined by

$$
P(\sigma, f)=\limsup _{n \rightarrow \infty} \frac{1}{n} \log \sum_{\sigma^{n} x=x} e^{f^{n}(x)}
$$

and if $\sigma$ is topologically mixing then the lim sup may be replaced with a limit.

We now consider skew product extensions of a shift of finite type $\sigma: \Sigma_{A}^{+} \rightarrow \Sigma_{A}^{+}$, which we will assume to be topologically mixing. Let $G$ be a countable group and let 
$\psi: \Sigma_{A}^{+} \rightarrow G$ be a function depending only on two co-ordinates, $\psi(x)=\psi\left(x_{0}, x_{1}\right)$. (One may consider more general $\psi$ but this set-up suffices for our needs.) This data defines a skew product extension $\widetilde{\sigma}: \Sigma_{A}^{+} \times G \rightarrow \Sigma_{A}^{+} \times G$ by $\widetilde{\sigma}(x, g)=(\sigma x, g \psi(x))$. For $n \geq 1$ define $\psi_{n}$ by

$$
\psi_{n}(x)=\psi(x) \psi(\sigma x) \cdots \psi\left(\sigma^{n-1} x\right)
$$

then $\tilde{\sigma}^{n}(x, g)=(x, g)$ if and only if $\sigma^{n} x=x$ and $\psi_{n}(x)=1_{G}$, where $1_{G}$ is the identity element in $G$.

The map $\widetilde{\sigma}: \Sigma_{A}^{+} \times G \rightarrow \Sigma_{A}^{+} \times \underset{\widetilde{A}}{G}$ is itself a countable state Markov shift with alphabet $S \times G$ and transition matrix $\widetilde{A}$ defined by $\widetilde{A}((i, g),(j, h))=1$ if $A(i, j)=1$ and $\psi(i, j)=g^{-1} h$ and $\widetilde{A}((i, g),(j, h))=0$ otherwise. Clearly, $\widetilde{A}$ is locally finite and so the topological transitivity and topological mixing of $\widetilde{\sigma}$ are equivalent to $\widetilde{A}$ being irreducible and aperiodic, respectively.

Let $f: \Sigma_{A}^{+} \rightarrow \mathbb{R}$ be Hölder continuous and define $\tilde{f}: \Sigma_{A}^{+} \times G \rightarrow \mathbb{R}$ by $\widetilde{f}(x, g)=$ $f(x)$; then $\widetilde{f}$ is locally Hölder continuous and its Gurevič pressure $P_{G}(\widetilde{\sigma}, \widetilde{f})$ is defined. In fact, it is easy to see that, due to the mixing of $\sigma$,

$$
P_{G}(\widetilde{\sigma}, \widetilde{f})=\limsup _{n \rightarrow \infty} \frac{1}{n} \log \sum_{\substack{\sigma^{n} x=x \\ \psi_{n}(x)=1_{G}}} e^{f^{n}(x)} .
$$

It is clear that $P_{G}(\widetilde{\sigma}, \widetilde{f}) \leq P(\sigma, f)$ and it is interesting to ask when equality holds. Stadlbauer has shown this depends only on the amenability of the group $G$, provided the skew product and the function $f$ satisfy appropriate symmetry conditions, which we now describe.

Suppose there is a fixed point free involution $\kappa: S \rightarrow S$ such that $A(\kappa j, \kappa i)=$ $A(i, j)$, for all $i, j \in S$. We say that the skew product $\widetilde{\sigma}: \Sigma_{A}^{+} \times G \rightarrow \Sigma_{A}^{+} \times G$ is symmetric (with respect to $\kappa$ ) if $\psi(\kappa j, \kappa i)=\psi(i, j)^{-1}$. A function $f: \Sigma_{A}^{+} \rightarrow \mathbb{R}$ is called weakly symmetric if, for all $n \geq 1$ and and all length $n$ cylinders $\left[z_{0}, z_{1}, \ldots, z_{n-1}\right]$, there exists $D_{n}>0$ such that $\lim _{n \rightarrow \infty} D_{n}^{1 / n}=1$ and

$$
\sup _{\substack{x \in\left[z_{0}, \ldots, z_{n-1}\right] \\ y \in\left[\kappa z_{n-1}, \ldots, \kappa z_{0}\right]}} \exp \left(f^{n}(x)-f^{n}(y)\right) \leq D_{n} .
$$

The following is the main result of Stadlbauer [30], restricted to the case where the base is a (finite state) subshift of finite type. We will use this in subsequent arguments. (More generally, Stadlbauer considers skew product expansions of countable state Markov shifts.)

Proposition 3.3 (Stadlbauer [30], Theorem 4.1 and Theorem 5.6) Let $\widetilde{\sigma}: \Sigma_{A}^{+} \times G \rightarrow$ $\Sigma_{A}^{+} \times G$ be a transitive symmetric skew-product extension of a mixing subshift of finite type $\sigma: \Sigma_{A}^{+} \rightarrow \Sigma_{A}^{+}$by a countable group $G$. Let $f: \Sigma_{A}^{+} \rightarrow \mathbb{R}$ be a weakly symmetric Hölder continuous function and define $\widetilde{f}: \Sigma_{A}^{+} \times G \rightarrow \mathbb{R}$ by $\widetilde{f}(x, g)=f(x)$. Then $P_{G}(\widetilde{\sigma}, \widetilde{f})=P(\sigma, f)$ if and only if $G$ is amenable. 
Remark 3.4 In [30], Stadlbauer considers skew products with $\psi$ depending on only one co-ordinate. However, replacing $S$ by $\mathcal{W}^{2}$, one can easily recover the above formulation.

We end this section by discussing two-sided subshifts of finite type and suspended flows over them. Given a finite alphabet $S$ and transition matrix $A$, we define

$$
\Sigma_{A}=\left\{x=\left(x_{n}\right)_{n=0}^{\infty} \in S^{\mathbb{Z}}: A\left(x_{n}, x_{n+1}\right)=1 \forall n \in \mathbb{Z}\right\}
$$

and the (two-sided) shift of finite type $\sigma: \Sigma_{A} \rightarrow \Sigma_{A}$ by $(\sigma x)_{n}=x_{n+1}$. As before, we give $\Sigma_{A}$ with the product topology induced by the discrete topology on $S$ and this is compatible with the metric $d(x, y)=2^{-n(x, y)}$, where

$$
n(x, y)=\inf \left\{|n|: x_{n} \neq y_{n}\right\}
$$

with $n(x, y)=\infty$ if $x=y$. Then $\Sigma_{A}$ is compact and $\sigma$ is a homeomorphism. There is an obvious one-to-one correspondence between the periodic points of $\sigma: \Sigma_{A} \rightarrow \Sigma_{A}$ and $\sigma: \Sigma_{A}^{+} \rightarrow \Sigma_{A}^{+}$. Furthermore, we may pass from Hölder functions on $\Sigma_{A}$ to Hölder functions on $\Sigma_{A}^{+}$in such a way that sums around periodic orbits are preserved. More precisely, we have the following lemma, due originally to Sinai.

Lemma 3.5 [20] Let $f: \Sigma_{A} \rightarrow \mathbb{R}$ be Hölder continuous. Then there is a Hölder continuous function $f^{\prime}: \Sigma_{A}^{+} \rightarrow \mathbb{R}$ (with a smaller Hölder exponent) such that $f^{n}(x)=\left(f^{\prime}\right)^{n}(x)$, whenever $\sigma^{n} x=x$.

We may also define suspended flows over $\sigma: \Sigma_{A} \rightarrow \Sigma_{A}$. Given a strictly positive continuous function $r: \Sigma_{A} \rightarrow \mathbb{R}^{+}$, we define the $r$-suspension space

$$
\Sigma_{A}^{r}=\left\{(x, s): x \in \Sigma_{A}, 0 \leq s \leq r(x)\right\} / \sim,
$$

where $(x, r(x)) \sim(\sigma x, 0)$. The suspended flow $\sigma_{t}^{r}: X_{A}^{r} \rightarrow X_{A}^{r}$ is defined by $\sigma_{t}^{r}(x, s)=(x, s+t)$ modulo the identifications. Clearly, there is a natural one-to-one correspondence between periodic orbits for $\sigma_{t}^{r}: \Sigma_{A}^{r} \rightarrow \Sigma_{A}^{r}$ and periodic orbits for $\sigma: \Sigma_{A} \rightarrow \Sigma_{A}$, and a $\sigma^{r}$-periodic orbit is prime if and only if the corresponding $\sigma$-periodic orbit is prime. Furthermore, if $\gamma$ is a closed $\sigma^{r}$-orbit corresponding to the closed $\sigma$-orbit $\left\{x, \sigma x, \ldots, \sigma^{n-1} x\right\}$ then the period of $\gamma$ is equal to $r^{n}(x)$.

\section{Coverings and geodesic flows}

As in the introduction, we shall write $M=X / \Gamma, \widetilde{M}=X / \Gamma_{0}$ and $G=\Gamma / \Gamma_{0}$. We always assume that $\Gamma_{0}$ is not the trivial group. (If it were then $G=\Gamma$ would not be amenable while $\delta\left(\Gamma_{0}\right)=h\left(X / \Gamma_{0}\right)=0$ so the conclusions of Theorem 1.1 hold automatically.) There is a natural dynamical system related to the geometry of $M$, namely the geodesic flow on the unit-tangent bundle $S M=\left\{(x, v) \in T M:\|v\|_{x}=\right.$ 1 , where $\|\cdot\|_{x}$ is the norm induced by the Riemannian structure on $T_{x} M$. For future reference, we write $p: S M \rightarrow M$ for the projection. The geodesic flow $\phi_{t}: S M \rightarrow$ 
$S M$ is defined as follows. Given $(x, v) \in S M$, there is a unique unit-speed geodesic $\gamma: \mathbb{R} \rightarrow M$ with $\gamma(0)=x$ and $\dot{\gamma}(0)=v$. We then define $\phi_{t}(x, v)=(\gamma(t), \dot{\gamma}(t))$. Since $M$ is geodesically complete, $\phi_{t}$ has no fixed points.

The non-wandering set $\Omega(\Gamma) \subset S M$ for the flow $\phi_{t}: S M \rightarrow S M$ is defined to be the set of points $x \in S M$ with the property that for every open neighbourhood $U$ of $x$, there exists $t>0$ such that $\phi_{t}(U) \cap U \neq \varnothing$. The set $\Omega(\Gamma)$ is compact and it is a subset of $\bigcup_{x \in C(\Gamma) / \Gamma} S_{x} M$. If $M$ is compact then $\Omega(\Gamma)=S M$ but if $M$ is not compact then $\Omega(\Gamma)$ has empty interior in $S M$.

The manifold $\widetilde{M}$ is a regular $G$-cover of $M$ and we let $\pi: \widetilde{M} \rightarrow M$ denote the covering map. The geodesic flow $\widetilde{\phi}_{t}: S \widetilde{M} \rightarrow S \widetilde{M}$ is defined in a similar way to the geodesic flow on $S M$ and also has no fixed points. We write $\Omega\left(\Gamma_{0}\right) \subset S \widetilde{M}$ for its nonwandering set which is not compact (as long as $G$ is infinite). We write $\widetilde{p}: S \widetilde{M} \rightarrow M$ for the projection. The action of $G$ extends to the unit-tangent bundle $S \widetilde{M}$ by the formula $g(x, v)=\left(g x, D g_{x} v\right)$ and induces a regular covering $\pi: S \widetilde{M} \rightarrow S M$ which commutes with the two flows. (The use of $\pi$ to denote both coverings should not cause any confusion.)

The restriction of both the geodesic flows to their non-wandering sets are example of a hyperbolic flows, where we say that a $C^{1}$ flow $\phi_{t}: \Omega \rightarrow \Omega$ is hyperbolic if

(1) there is a continuous $D \phi$-invariant splitting of the tangent bundle

$$
T_{\Omega}(S M)=E^{0} \oplus E^{s} \oplus E^{u},
$$

where $E^{0}$ is the line bundle tangent to the flow and where there exists constants $C, c>0$ such that

(i) $\left\|D \phi_{t} v\right\| \leq C e^{-c t}\|v\|$, for all $v \in E^{s}$ and $t>0$;

(ii) $\left\|D \phi_{-t} v\right\| \leq C e^{-c t}\|v\|$, for all $v \in E^{u}$ and $t>0$,

(2) $\phi_{t}: \Omega \rightarrow \Omega$ is transitive (i.e. it has a dense orbit),

(3) the periodic $\phi$-orbits are dense in $\Omega$, and

(4) there is an open set $U \supset \Omega$ such that $\Omega=\bigcap_{t \in \mathbb{R}} \phi_{t}(U)$.

This is due to Anosov [1] when $M$ is compact and Eberlein [9] in general. However, a crucial difference between the flows is the compactness of $\Omega(\Gamma)$. This will allow a symbolic coding of $\phi_{t}: \Omega(\Gamma) \rightarrow \Omega(\Gamma)$, as we shall discuss in the next section.

There is a natural one-to-one correspondence between (prime) periodic orbits for $\phi_{t}: \Omega(\Gamma) \rightarrow \Omega(\Gamma)$ and (prime) closed geodesics on $M$, with the least period being equal to the length of the closed geodesic. We will typically write $\gamma$ for either a closed geodesic or a periodic orbit and allow the context to distinguish them. We will write $l(\gamma)$ for the length (period) of $\gamma$. The number $h=h(M)$ defined in the introduction as the exponential growth rate of the number of $\gamma$ with $l(\gamma) \leq T$ is also equal to the topological entropy of $\phi[3]$.

\section{Markov sections and symbolic dynamics}

A particularly useful aspect of hyperbolic flows on compact invariant sets is that they admit a description by finite state symbolic dynamics. We shall outline this construction below for the geodesic flow $\phi_{t}: \Omega(\Gamma) \rightarrow \Omega(\Gamma)$. 
Consider the unrestricted geodesic flow $\phi_{t}: S M \rightarrow S M$. Given $x \in \Omega(\Gamma)$ and $\epsilon>0$, we define the (strong) local stable manifold $W_{\epsilon}^{s}(x)$ and (strong) local unstable manifold $W_{\epsilon}^{u}(x)$ for a point $x \in S M$ by

$$
W_{\epsilon}^{s}(x)=\left\{y \in S M: d\left(\phi_{t}(x), \phi_{t}(y)\right) \leq \epsilon \forall t \geq 0 \text { and } \lim _{t \rightarrow \infty} d\left(\phi_{t}(x), \phi_{t}(y)\right)=0\right\}
$$

and

$$
\begin{aligned}
& W_{\epsilon}^{u}(x) \\
& =\left\{y \in S M: d\left(\phi_{-t}(x), \phi_{-t}(y)\right) \leq \epsilon \forall t \geq 0 \text { and } \lim _{t \rightarrow \infty} d\left(\phi_{-t}(x), \phi_{-t}(y)\right)=0\right\} .
\end{aligned}
$$

Provided $\epsilon>0$ is sufficiently small, these sets are diffeomorphic to ( $\operatorname{dim} M-1)$ dimensional embedded disks. If $x$ and $y$ are sufficiently close then there is a unique $t \in[-\epsilon, \epsilon]$ such that $W_{\epsilon}^{s}(x) \cap W_{\epsilon}^{u}\left(\phi_{t}(y)\right) \neq \varnothing$ and, furthermore, this intersection consists of a single point denoted $[x, y]$. This pairing $[\cdot, \cdot]$ is called the local product structure.

Let $D_{1}, \ldots, D_{k}$ be a family of co-dimension one disks that form a local cross section to the flow and let $\mathcal{P}$ denote the Poincaré map between them. For each $i=1, \ldots, k$ and small $\delta>0$, there is a natural projection map

$$
\operatorname{pr}_{D_{i}}:\left\{\phi_{t} x: x \in D_{i},-\delta \leq t \leq \delta\right\} \rightarrow D_{i}
$$

given by $\operatorname{pr}_{D_{i}}\left(\phi_{t} x\right)=x$. For each $i=1, \ldots, k$, let $T_{i} \subset \operatorname{int}\left(D_{i}\right) \cap \Omega(\Gamma)$ be sets which are chosen to be rectangles in the sense that whenever $x, y \in T_{i}$ then $[x, y]_{D_{i}}:=$ $\operatorname{pr}_{D_{i}}([x, y]) \in T_{i}$ and proper (i.e. $T_{i}=\overline{\operatorname{int}\left(T_{i}\right)}$ for each $i$ ). (Here and subsequently, the interiors are taken relative to $D_{i} \cap \Omega(\Gamma)$.) We then say that $T_{1}, \ldots, T_{k}$ are Markov sections for the flow if

(1) for $x \in \operatorname{int}\left(T_{i}\right)$ with $\mathcal{P} \in \operatorname{int}\left(T_{j}\right)$ then $\mathcal{P}\left(W^{s}\left(x, T_{i}\right)\right) \subset W^{s}\left(\mathcal{P} x, T_{j}\right)$, and

(2) for $x \in \operatorname{int}\left(T_{i}\right)$ with $\mathcal{P}^{-1} x \in \operatorname{int}\left(T_{j}\right)$ then $\mathcal{P}^{-1}\left(W^{u}\left(x, T_{i}\right)\right) \subset W^{u}\left(\mathcal{P}^{-1} x, T_{j}\right)$,

where $W^{s}\left(x, T_{i}\right)$ and $W^{u}\left(x, T_{i}\right)$ denote the projections of $W_{\epsilon}^{s}(x)$ and $W_{\epsilon}^{u}(x)$ onto $T_{i}$, respectively.

Proposition 5.1 (Bowen [4]) For all $\epsilon>0$, the flow $\phi_{t}: \Omega(\Gamma) \rightarrow \Omega(\Gamma)$ has Markov sections $T_{1}, \ldots, T_{k}$ such that $\operatorname{diam}\left(T_{i}\right)<\epsilon$, for $i=1, \ldots, k$ and such that $\bigcup_{i=1}^{k} \phi_{[0, \epsilon]} T_{i}=\Omega(\Gamma)$. flow.

These sections may be chosen to reflect the time-reversal symmetry of the geodesic

Lemma 5.2 (Adachi [2], Rees [25]) The Markov sections $T_{1}, \ldots, T_{k}$ may be chosen so that there is a fixed point free involution $\kappa:\{1, \ldots, k\} \rightarrow\{1, \ldots, k\}$ such that $A(\kappa j, \kappa i)=1$ if and only if $A(i, j)=1$. Furthermore, the involution is consistent with the time reversing involution: $T_{\kappa i}=\iota\left(T_{i}\right)$, where $\iota: S M \rightarrow S M$ is the map $\iota(x, v)=(x,-v)$. 
The Markov sections allow us to relate $\phi_{t}: \Omega(\Gamma) \rightarrow \Omega(\Gamma)$ to a suspended flow over a mixing subshift of finite type, whose alphabet $\{1, \ldots, k\}$ corresponds to the Markov sections $\left\{T_{1}, \ldots, T_{k}\right\}$.

Proposition 5.3 (Bowen [4]) There exists a mixing subshift of finite type $\sigma: \Sigma_{A} \rightarrow$ $\Sigma_{A}$, a strictly positive Hölder continuous function $r: \Sigma_{A} \rightarrow \mathbb{R}^{+}$and a map $\vartheta$ : $\Sigma_{A}^{r} \rightarrow \Omega(\Gamma)$ such that

(1) $\vartheta$ is a semi-conjugacy (i.e. $\phi_{t} \circ \vartheta=\vartheta \circ \sigma_{t}^{r}$ );

(2) $\vartheta$ is a surjection and is one-to-one on a residual set;

(3) $h(\phi)=h\left(\sigma^{r}\right)=h$.

The above coding does not give a one-to-one correspondence between periodic orbits for $\phi$ and $\sigma^{r}$. This is overcome by the following result, which is Bowen's generalisation to flows of a result of Manning for diffeomorphisms [16]. For a flow $\xi_{t}$, let $v(\xi, T)$ denote the number of prime periodic $\xi$-orbits of period $T$ and $N_{\xi}(T)$ the number of periodic $\xi$-orbits to period at most $T$.

Lemma 5.4 (Bowen [4]) There exist a finite number of subshifts of finite type $\sigma_{j}$ : $\Sigma_{j} \rightarrow \Sigma_{j}$ and strictly positive Hölder continuous functions $r_{j}: \Sigma_{j} \rightarrow \mathbb{R}^{+}, j=$ $1, \ldots, q$, such that

(1) $h\left(\sigma^{r_{j}}\right)<h, j=1, \ldots, q$;

(2)

$$
v(\phi, T)=v\left(\sigma^{r}, T\right)+\sum_{j=1}^{q}(-1)^{\eta_{j}} v\left(\sigma_{i}^{r_{j}}, T\right),
$$

where $\eta_{j} \in\{0,1\}, j=1, \ldots, q$.

Corollary $5.5 N_{\phi}(T)=N_{\sigma^{r}}(T)+O\left(e^{h^{\prime} T}\right)$, where $h^{\prime}:=\max _{1 \leq j \leq q} h\left(\sigma^{r_{j}}\right)<h$.

We end the section by noting the following result.

Lemma 5.6 [20] The entropy $h$ is the unique real number for which $P(\sigma,-h r)=0$.

\section{The skew product extension}

In this section we will describe a skew product extension of (the one-sided version of) the shift of finite type introduced above, which will serve to encode information about how orbits on $S M$ lift to $S \widetilde{M}$, and relate this construction to the result of Stadlbauer, Proposition 3.3, stated above.

Choose $\epsilon_{0}>0$ sufficiently small that every open ball in $S M$ with diameter less than $\epsilon_{0}$ is simply connected. Let $U \subset S M$ be such an open ball. Then $\pi^{-1}(U)=$ $\bigcup_{g \in G} g \cdot \widetilde{U}$, where $\widetilde{U}$ is a connected component of $\pi^{-1}(U)$. Since we can choose the Markov sections $T_{i}$ to have arbitrarily small diameters, for each $i=1, \ldots, k$, we can choose $U_{i}$ be an open ball of diameter less than $\epsilon_{0}$ containing $T_{i}$. As above, we may 
write $\pi^{-1}\left(U_{i}\right)=\bigcup_{g \in G} g \cdot \widetilde{U}_{i}$ and $\pi^{-1}\left(T_{i}\right)=\bigcup_{g \in G} g \cdot \widetilde{T}_{i}$, where $\widetilde{T}_{i}=\pi^{-1}\left(T_{i}\right) \cap \widetilde{U}_{i}$, and we may assume that this decomposition is chosen with $\widetilde{\iota}\left(\widetilde{T}_{i}\right)=\widetilde{T}_{\kappa i}$, where $\widetilde{\iota}$ is the time-reversing involution $\widetilde{\imath}: S \widetilde{M} \rightarrow S \widetilde{M}$ given by $\widetilde{\imath}(x, v)=(x,-v)$.

We will write $\mathcal{T}=\left\{T_{1}, \ldots, T_{k}\right\}$ and use the notation

$$
\widetilde{\mathcal{T}}=\pi^{-1}(\mathcal{T})=\bigcup_{i=1}^{k} \bigcup_{g \in G} g \cdot \widetilde{T}_{i} .
$$

Notice that each lifted section $g \cdot \widetilde{T}_{i}$ is transverse to the flow $\widetilde{\phi}_{t}: S \widetilde{M} \rightarrow S \widetilde{M}$. We write $\widetilde{\mathcal{P}}: \widetilde{\mathcal{T}} \rightarrow \widetilde{\mathcal{T}}$ for the Poincaré map.

Lemma 6.1 Suppose that $A(i, j)=1$. There is a unique $g=g(i, j) \in G$ such that for any $x \in T_{i} \cap \mathcal{P}^{-1}\left(T_{j}\right)$, any $\widetilde{x} \in \pi^{-1}(x)$, and any $h \in G$, if $\widetilde{x} \in h \cdot \widetilde{T}_{i}$ then $\widetilde{\mathcal{P}}(\widetilde{x}) \in h g \cdot \widetilde{T}_{j}$. In addition, $g(\kappa j, \kappa i)=g(i, j)^{-1}$.

Proof We will begin be proving the existence and uniqueness of $g$. Let $x_{1}, x_{2} \in$ $T_{i} \cap \mathcal{P}^{-1}\left(T_{j}\right)$ and let $c_{1}$ and $c_{2}$ be the $\phi$-orbit segments from $c_{1}(0)=x_{1}$ and $c_{2}(0)=x_{2}$ to $c_{1}(1)=\mathcal{P}\left(x_{1}\right)$ and $c_{2}(1)=\mathcal{P}\left(x_{2}\right)$. We will show that there is a unique $g \in G$ such that the unique lifts of $c_{1}$ and $c_{2}$ that begin in $\widetilde{T}_{i}$ both have terminal points in $g \cdot \widetilde{T}_{j}$. The statement for all $h \in G$ will follow by translating by the isometry $h \in G$.

Let $\widetilde{c}_{1}$ and $\widetilde{c}_{2}$ be lifts of $c_{1}$ and $c_{2}$ with $\widetilde{c}_{1}(0), \widetilde{c}_{2}(0) \in \widetilde{T}_{i}$. Having chosen the Markov partition to have sufficiently small diameters and the flow times between rectangles to be sufficiently small, there is an open ball $U \subset S M$ of diameter less than $\epsilon_{0}$ containing $T_{i}, T_{j}, c_{1}$ and $c_{2}$. Let $\widetilde{U}$ be the connected component of $\pi^{-1}(U)$ containing $\widetilde{c}_{1}$. Then $\widetilde{U} \cap \widetilde{T}_{i} \neq \varnothing$ and $\widetilde{U} \cap g \cdot \widetilde{T}_{j} \neq \varnothing$ and so $\widetilde{T}_{i} \cup g \cdot \widetilde{T}_{j} \subseteq \widetilde{U}$. It follows that $\widetilde{c}_{2}$ is entirely contained in $\widetilde{U}$ and so we must have $\widetilde{c}_{2}(1) \in g \cdot \widetilde{T}_{j}$ as required.

For the final part, we note that $\iota\left(\widetilde{c}_{1}\right)$ is an orbit segment from $g \cdot \widetilde{T}_{\kappa j}$ to $\widetilde{T}_{\kappa i}$. It follows from the uniqueness in the previous that $g(\kappa j, \kappa i)=g(i, j)^{-1}$.

We use the preceding lemma to define a skew product extension of the one-sided shift of finite type $\sigma: \Sigma_{A}^{+} \rightarrow \Sigma_{A}^{+}$. Define $\psi: \Sigma_{A}^{+} \rightarrow G$ (depending on two coordinates) by $\psi(x)=\psi\left(x_{0}, x_{1}\right)=g\left(x_{0}, x_{1}\right)$, where $g=g\left(x_{0}, x_{1}\right)$ is the unique element of $G$ given by Lemma 6.1. Then the skew product $\widetilde{\sigma}: \Sigma_{A}^{+} \times G \rightarrow \Sigma_{A}^{+} \times G$ is defined by

$$
\widetilde{\sigma}(x, g)=(\sigma x, g \psi(x)) .
$$

Furthermore, part (2) of Lemma 6.1 shows that the skew product extension is symmetric (with respect to the involution $\kappa)$, i.e. that $\psi(\kappa j, \kappa i)=\psi(i, j)^{-1}$.

We note the relationship between periodic orbits for the lifted geodesic flow and for the skew product.

Lemma 6.2 A periodic $\phi$-orbit $\gamma$ in $S M$, corresponding to a periodic $\sigma$-orbit $\tau=$ $\left\{x, \sigma x, \ldots, \sigma^{n-1} x\right\}$, lifts to a periodic orbit on $S \widetilde{M}$ if and only if $\psi_{n}(x)=1_{G}$.

Proof We will treat $\vartheta(x)$ as the initial point on $\gamma$. Let $\tilde{\gamma}$ be the lift of $\gamma$ which starts in $\widetilde{T}_{x_{0}}$. By Lemma 6.1, $\widetilde{\gamma}$ ends in $\psi_{n}(x) \cdot \widetilde{T}_{x_{0}}$ and is thus periodic if and only if $\psi_{n}(x)=1_{G}$. 
We shall apply Proposition 3.3 to the skew product extension $\widetilde{\sigma}: \Sigma_{A}^{+} \times G \rightarrow$ $\Sigma_{A}^{+} \times G$. To do this, we need to establish that two further conditions are satisfied: that $\tilde{\sigma}$ is transitive and that $r$ is weakly symmetric. We start with transitivity.

Lemma 6.3 If $G$ is not equal to $\pi_{1}(M)$ then the map $\tilde{\sigma}: \Sigma_{A}^{+} \times G \rightarrow \Sigma_{A}^{+} \times G$ is transitive.

Proof If $G$ is not equal to $\pi_{1}(M)$ then the geodesic flow $\widetilde{\phi}_{t}: \Omega\left(\Gamma_{0}\right) \rightarrow \Omega\left(\Gamma_{0}\right)$ is transitive [9]. Let $x \in \Omega\left(\Gamma_{0}\right)$ be a point with dense $\widetilde{\phi}$-orbit. Without loss of generality $x \in \widetilde{\mathcal{T}}$ and then $\left\{\widetilde{\mathcal{P}}^{n} x\right\}_{n=-\infty}^{\infty}$ is dense in $\widetilde{\mathcal{T}}$. Suppose that $\widetilde{A}\left(\left(i_{j}, g_{j}\right),\left(i_{j+1}, g_{j+1}\right)\right)=1$, where $\widetilde{A}$ is the transition matrix for $\Sigma_{A}^{+} \times G$, for $j=0, \ldots, n$. Then

$$
U=\bigcap_{j=0}^{n} \widetilde{\mathcal{P}}^{-j}\left(\operatorname{int}\left(g_{j} \cdot \widetilde{T}_{i_{j}}\right)\right)
$$

is non-empty and open in $\widetilde{\mathcal{T}}$. (Here $\operatorname{int}\left(g_{j} \cdot \widetilde{T}_{i_{j}}\right)$ is taken with respect to the intersection of the co-dimension one disk containing $g_{j} \cdot \widetilde{T}_{i j}$ with $\Omega\left(\Gamma_{0}\right)$.) Since $x$ has dense $\widetilde{\mathcal{P}}$ orbit, $\widetilde{\mathcal{P}}^{m} x \in U$ for some $m \in \mathbb{Z}$. Then $\widetilde{\mathcal{P}}^{m+j}(x) \in \operatorname{int}\left(g_{j} \cdot \widetilde{T}_{i_{j}}\right)$ for $j=0, \ldots, n$. By definition, this implies that the $\widetilde{\sigma}$-orbit of $\left(\vartheta(\pi(x)), g_{0}\right) \in \Sigma_{A}^{+} \times G$ (where $\vartheta(\pi(x))$ is identified with a point in the one-sided shift) passes through the (arbitrary) cylinder $\left[\left(i_{0}, g_{0}\right), \ldots,\left(i_{n}, g_{n}\right)\right]$ and is thus dense in $\Sigma_{A}^{+} \times G$. Therefore, $\widetilde{\sigma}: \Sigma_{A}^{+} \times G \rightarrow \Sigma_{A}^{+} \times G$ is transitive.

Let $r: \Sigma_{A} \rightarrow \mathbb{R}$ be the Hölder continuous function defined by Proposition 5.3. By Lemma 3.5, there is a Hölder continuous function on $\Sigma_{A}^{+}$, which we will abuse notation by continuing to call $r$, with the same sums around periodic orbits.

Lemma 6.4 For any $\xi \in \mathbb{R}$, the function $-\xi r: \Sigma_{A}^{+} \rightarrow \mathbb{R}$ is weakly symmetric.

Proof It suffices to show that $r$ is weakly symmetric. Since $\sigma: \Sigma_{A}^{+} \rightarrow \Sigma_{A}^{+}$is mixing, there exists $N \geq 0$ such that, for each length $n$ cylinder $\left[z_{0}, \ldots, z_{n-1}\right]$, we may find a periodic point $x \in\left[z_{0}, \ldots, z_{n-1}\right]$ of period $n+N$. Writing $x=$ $\left(x_{0}, \ldots, x_{n+N-1}, x_{0}, \ldots\right)$, we set $\kappa x=\left(\kappa x_{n+N-1}, \ldots, \kappa x_{0}, \kappa x_{n+N-1}, \ldots\right)$. Clearly, $\sigma^{N}(\kappa x)$ is a periodic point of period $n+N$ and $\sigma^{N}(\kappa x) \in\left[\kappa z_{n-1}, \ldots, \kappa z_{0}\right]$. Furthermore, $r^{n+N}(x)=l(\gamma)$, for some $\phi$-periodic orbit $\gamma$ and

$$
r^{n+N}\left(\sigma^{N}(\kappa x)\right)=r^{n+N}(\kappa x)=l(\iota \gamma)=l(\gamma),
$$

where $\iota \gamma$ is the time-reversed periodic orbit corresponding to $\gamma$. We therefore have

$$
\begin{aligned}
\exp \left(r^{n}(x)-r^{n}\left(\sigma^{N}(\kappa x)\right)\right) & =\exp \left(\left(l(\gamma)-r^{N}\left(\sigma^{n} x\right)\right)-\left(l(\gamma)-r^{N}\left(\sigma^{n+N}(\kappa x)\right)\right)\right) \\
& =\exp \left(r^{N}\left(\sigma^{n+N}(\kappa x)\right)-r^{N}\left(\sigma^{n} x\right)\right) \leq \exp \left(2 N\|r\|_{\infty}\right),
\end{aligned}
$$

for some constant $C>0$. 
Now let $x^{\prime} \in\left[z_{0}, \ldots, z_{n-1}\right]$ and $y^{\prime} \in\left[\kappa z_{n-1}, \ldots, \kappa z_{0}\right]$ be arbitrary. We have

$$
\begin{aligned}
\exp \left(r^{n}\left(x^{\prime}\right)-r^{n}\left(y^{\prime}\right)\right) & =\exp \left(r^{n}(x)-r^{n}\left(\sigma^{N}(\kappa x)\right)\right) \frac{\exp \left(r^{n}\left(x^{\prime}\right)-r^{n}(x)\right)}{\exp \left(r^{n}\left(y^{\prime}\right)-r^{n}\left(\sigma^{N}(\kappa x)\right)\right)} \\
& \leq \exp (N C) \exp \left(2 c /\left(1-2^{-\alpha}\right)\right),
\end{aligned}
$$

where $r$ satisfies the Hölder condition $|r(x)-r(y)| \leq c d(x, y)^{\alpha}$. This completes the proof.

\section{Zeta functions}

In this section we shall prove that the equality of $h=h(M)$ and $h(\widetilde{M})$ is equivalent to amenability of $G$. To do this, we need to relate the growth of closed geodesics in $\mathcal{C}(\widetilde{M}, W)$ or, equivalently, of periodic $\widetilde{\phi}$-orbits which have non-empty intersection with $W$, to the Gurevič pressure. We will make particular choices of $W$. First, for each $i=1, \ldots, k$, we let $\widetilde{V}_{i}$ denote the interior of $\left\{\widetilde{\phi}_{t}(x): x \in \widetilde{T}_{i},-\epsilon \leq t \leq \epsilon\right\}$ in $\Omega\left(\Gamma_{0}\right)$, where $0<\epsilon \leq(\inf r) / 3$. Now choose an open relatively compact set $W_{i}^{(1)} \subset S \widetilde{M}$ such that $\widetilde{V}_{i}=W_{i}^{(1)} \cap \Omega\left(\Gamma_{0}\right)$ and set

$$
W^{(1)}=\bigcup_{i=1}^{k} W_{i}^{(1)}
$$

We also define

$$
W^{(2)}=\bigcup_{i=1}^{k} \operatorname{int}\left(\left\{\widetilde{\phi}_{t}(x): x \in \widetilde{D}_{i},-2 \epsilon \leq t \leq 2 \epsilon\right\}\right),
$$

where $\widetilde{D}_{i}$ is the component of $\pi^{-1}\left(D_{i}\right)$ containing $\widetilde{T}_{i}$.

For each $W \subset S M$ (not necessarily open), we define a zeta function, analogous to the usual zeta function for a flow but associated to $\mathcal{C}(\widetilde{M}, W)$, by

$$
\zeta_{W}(s)=\prod_{\gamma \in \mathcal{C}^{\prime}(\widetilde{M}, W)}\left(1-e^{-s l(\gamma)}\right)^{-1}=\exp \sum_{m=1}^{\infty} \sum_{\gamma \in \mathcal{C}^{\prime}(\widetilde{M}, W)} \frac{e^{-s m l(\gamma)}}{m},
$$

where $\mathcal{C}^{\prime}(\tilde{M}, W)$ denotes the prime closed geodesics in $\mathcal{C}(\tilde{M}, W)$. The zeta functions $\zeta_{W^{(1)}}(s)$ and $\zeta_{W^{(2)}}(s)$ both have abscissa of convergence $\widetilde{h}:=h(\widetilde{M})$. Since

$$
W^{(1)} \cap \Omega\left(\Gamma_{0}\right) \subset W:=\bigcup_{i=1}^{k}\left\{\widetilde{\phi}_{t}(x): x \in \widetilde{T}_{i},-\epsilon \leq t \leq \epsilon\right\} \subset W^{(2)},
$$

we have that $\zeta(s):=\zeta_{W}(s)$ also has abscissa of convergence $\widetilde{h}$.

A similar function may be defined using the set $\mathcal{P}^{\prime}$ of prime periodic $\widetilde{\sigma}$-orbits which intersect $\Sigma_{A}^{+} \times\left\{1_{G}\right\}$ : 


$$
Z(s)=\prod_{\tau \in \mathcal{P}^{\prime}}\left(1-e^{-s \lambda(\tau)}\right)^{-1}
$$

where, for $\tau=\left\{(x, g), \widetilde{\sigma}(x, g), \ldots, \tilde{\sigma}^{n-1}(x, g)\right\}, \lambda(\tau)=r^{n}(x)$. (One can, of course, describe this in terms of a suspended semi-flow over $\Sigma_{A}^{+} \times G$ but this would make the notation more cumbersome.) A standard calculation gives

$$
Z(s)=\exp \sum_{n=1}^{\infty} \frac{1}{n} \sum_{(x, g) \in \mathcal{P}_{n}} e^{-s r^{n}(x)}
$$

where

$\mathcal{P}_{n}=\left\{(x, g): \widetilde{\sigma}^{n}(x, g)=(x, g)\right.$ and $\widetilde{\sigma}^{m}(x, g) \in \Sigma_{A}^{+} \times\left\{1_{G}\right\} \quad$ for some $\left.0 \leq m<n\right\}$.

It is this last function that will be related to Gurevič pressure.

The next lemma follows immediately from Lemma 5.4. In particular, the discrepancy between the number of periodic $\widetilde{\phi}$-orbits with $l(\gamma) \leq T$ and the number of periodic $\widetilde{\sigma}$-orbits with $\lambda(\tau)=r^{n}(x) \leq T$ is at worst $O\left(e^{h^{\prime} T}\right)$.

Lemma 7.1 $\zeta(s) / Z(s)$ is analytic and non-zero for $\operatorname{Re}(s)>h^{\prime}$.

Corollary 7.2 $\zeta(s)$ has abscissa of convergence $h$ if and only if $Z(s)$ has abscissa of convergence $h$.

Let

$$
\mathcal{Q}_{n}=\left\{x: \sigma^{n} x=x \text { and } \psi_{n}(x)=1_{G}\right\} .
$$

Lemma 7.3 For all $n \geq 1, \# \mathcal{Q}_{n} \leq \# \mathcal{P}_{n} \leq n \# \mathcal{Q}_{n}$. Hence, for $s \in \mathbb{R}$,

$$
\sum_{\substack{\sigma^{n} x=x \\ \psi_{n}(x)=1_{G}}} e^{-s r^{n}(x)} \leq \sum_{\substack{\tilde{\sigma}^{n}(x, g)=(x, g) \\ \exists 0 \leq m<n: \widetilde{\sigma}^{m}(x, g) \in \Sigma_{A}^{+} \times\left\{1_{G}\right\}}} e^{-s r^{n}(x)} \leq n \sum_{\substack{\sigma^{n} x=x \\ \psi_{n}(x)=1_{G}}} e^{-s r^{n}(x)} .
$$

Proof Since $\tilde{\sigma}^{n}(x, g)=(x, g)$ if and only if $\sigma^{n} x=x$ and $\psi_{n}(x)=1_{G}$, the first inequality follows by considering the the injection $\mathcal{Q}_{n} \rightarrow \mathcal{P}_{n}: x \mapsto\left(x, 1_{G}\right)$. On the other hand, when $\tilde{\sigma}^{n}(x, g)=(x, g)$, the condition $\widetilde{\sigma}^{m}(x, g) \in \Sigma_{A}^{+} \times\left\{1_{G}\right\}$ is equivalent to $\psi_{m}(x) g=1_{G}$, i.e. $g=\psi_{m}(x)^{-1}$. So, for each $\sigma^{n} x=x$ with $\psi_{n}(x)=1$, $\left\{g \in G:(x, g) \in \mathcal{P}_{n}\right\} \subset\left\{1, \psi(x)^{-1}, \ldots, \psi_{n-1}(x)^{-1}\right\}$ and hence has cardinality at most $n$. This proves the second inequality.

Now, as promised, we relate the abscissa of convergence of $Z(s)$ to Gurevič pressure.

Lemma 7.4 The abscissa of convergence of $Z(s)$ is the unique real number $\xi$ for which $P_{G}(\widetilde{\sigma},-\xi r)=0$. 
Proof It follows from Lemma 7.3 that

$$
\sum_{x \in \mathcal{Q}_{n}} e^{-s r^{n}(x)} \leq \sum_{(x, g) \in \mathcal{P}_{n}} e^{-s r^{n}(x)} \leq n \sum_{x \in \mathcal{Q}_{n}} e^{-s r^{n}(x)}
$$

and hence that

$$
\limsup _{n \rightarrow \infty} \frac{1}{n} \log \sum_{(x, g) \in \mathcal{P}_{n}} e^{-s r^{n}(x)}=\limsup _{n \rightarrow \infty} \frac{1}{n} \log \sum_{x \in \mathcal{Q}_{n}} e^{-s r^{n}(x)} .
$$

Since

$$
P_{G}(\widetilde{\sigma},-\xi r)=\limsup _{n \rightarrow \infty} \frac{1}{n} \log \sum_{x \in \mathcal{Q}_{n}} e^{-\xi r^{n}(x)},
$$

we have that $Z(\xi)$ converges if $P_{G}(\widetilde{\sigma},-\xi r)<0$ and diverges if $P_{G}(\widetilde{\sigma},-\xi r)>0$.

By Theorem 1 of [28] and the estimate $P_{G}(\widetilde{\sigma},-\xi r) \leq P(\sigma,-\xi r)$, the Gurevic pressure $P_{G}(\widetilde{\sigma},-\xi r)$ is always finite. Furthermore, $\xi \mapsto P_{G}(\widetilde{\sigma},-\xi r)$ is convex and hence continuous ([29, Proposition 4.4]). Choose $\xi, \xi^{\prime} \in \mathbb{R}$ with $\xi<\xi^{\prime}$. Write $r_{0}=\inf \left\{r^{n}(x) / n: \sigma^{n} x=x, n \geq 1\right\}$. Since $r: \Sigma_{A}^{+} \rightarrow \mathbb{R}$ is chosen to have the same sums around periodic orbits as the strictly positive function $r: \Sigma_{A} \rightarrow \mathbb{R}$, we have $r^{n}(x) \geq n \inf \left\{r(x): x \in \Sigma_{A}\right\}$ and $\inf \left\{r(x): x \in \Sigma_{A}\right\}>0$. Thus, $r_{0}>0$. Then, for $\sigma^{n} x=x, e^{-\xi^{\prime} r^{n}(x)} \leq e^{-\xi r^{n}(x)} e^{-n\left(\xi^{\prime}-\xi\right) r_{0}}$. Thus

$$
\begin{aligned}
P_{G}\left(\widetilde{\sigma},-\xi^{\prime} r\right) & =\limsup _{n \rightarrow \infty} \frac{1}{n} \log \sum_{x \in \mathcal{Q}_{n}} e^{-\xi^{\prime} r^{n}(x)} \\
& \leq \limsup _{n \rightarrow \infty} \frac{1}{n} \log \left(e^{-n\left(\xi^{\prime}-\xi\right) r_{0}} \sum_{x \in \mathcal{Q}_{n}} e^{-\xi r^{n}(x)}\right) \\
& =-\left(\xi^{\prime}-\xi\right) r_{0}+P_{G}(\widetilde{\sigma},-\xi r)<P_{G}(\widetilde{\sigma},-\xi r),
\end{aligned}
$$

so that $P_{G}(\widetilde{\sigma},-\xi r)$ is a strictly decreasing function. Also, $\lim _{\xi \rightarrow-\infty} P_{G}(\widetilde{\sigma}$, $-\xi r)=+\infty$ (from the trivial lower bound $P_{G}(\widetilde{\sigma},-\xi r) \geq-\xi\|r\|_{\infty}$ ) and $\lim _{\xi \rightarrow+\infty} P_{G}(\widetilde{\sigma},-\xi r)=-\infty$ (from $P_{G}(\widetilde{\sigma},-\xi r) \leq P(\sigma,-\xi r)$ ). Hence there is a unique $\xi \in \mathbb{R}$ such that $P_{G}(\widetilde{\sigma},-\xi r)=0$. By the above characterisation, this is the abscissa of convergence of $Z(s)$.

We may now prove our main result, formulated for closed geodesics.

Theorem 7.5 Let $\Gamma$ be a convex co-compact group of isometries of a pinched Hadamard manifold $X$ and let $\Gamma_{0}$ be a normal subgroup of $\Gamma$. Then $h\left(X / \Gamma_{0}\right)=$ $h(X / \Gamma)$ if and only if $G=\Gamma / \Gamma_{0}$ is amenable.

Proof By Lemma 5.6, we have $P(\sigma,-h r)=0$ and, by Proposition 3.3, $P_{G}(\widetilde{\sigma},-h r)<$ $P(\sigma,-h r)$ unless $G$ is amenable, in which case equality holds. Hence, if $G$ is amenable then $P(\widetilde{\sigma},-h r)=0$ and so $\widetilde{h}=h$. On the other hand, if $G$ is not amenable then $P_{G}(\widetilde{\sigma},-\xi r)=0$ for some $\xi<h$ and so, by Corollary 7.2 and Lemma $7.4, \widetilde{h}<h$. 
Remark 7.6 We could also have proved that equality of critical exponents implies amenability directly by replacing Stadlbauer's result with a recent result of Jaerisch [13], in which the Gurevič pressure is replaced by the logarithm of the spectral radius of a transfer operator associated to $\widetilde{\sigma}$ acting on a suitably chosen Banach space, together with some approximation arguments along the lines of those used in [23,24].

Open Access This article is distributed under the terms of the Creative Commons Attribution 4.0 International License (http://creativecommons.org/licenses/by/4.0/), which permits unrestricted use, distribution, and reproduction in any medium, provided you give appropriate credit to the original author(s) and the source, provide a link to the Creative Commons license, and indicate if changes were made.

\section{References}

1. Anosov, D.: Geodesic flows on closed Riemannian manifolds with negative curvature. In: Proceedings of the Steklov Institute of Mathematics, vol. 90. American Mathematical Society, Providence (1969)

2. Adachi, T.: Markov families for Anosov action with an involutive action. Nagoya Math. J. 104, 55-62 (1986)

3. Bowen, R.: Periodic orbits for hyperbolic flows. Am. J. Math. 94, 1-30 (1972)

4. Bowen, R.: Symbolic dynamics for hyperbolic flows. Am. J. Math. 95, 429-460 (1973)

5. Brooks, R.: The fundamental group an the spectrum of the Laplacian. Comment. Math. Helv. 56, 581-598 (1981)

6. Brooks, R.: The bottom of the spectrum of a Riemannian covering. J. Reine Angew. Math. 357, 101-114 (1985)

7. Burger, M.: Estimation de petite valeurs propres du laplacien d'un revêtement de variétés riemanniennes compactes. C. R. Acad. Sci. Paris Sér. I 302, 191-194 (1986)

8. Cohen, J.: Cogrowth and amenability in discrete groups. J. Funct. Anal. 48, 301-309 (1982)

9. Eberlein, P.: Geodesic flows on negatively curved manifolds I. Ann. Math. 95, 492-510 (1972)

10. Følner, E.: On groups with full Banach mean value. Math. Scand. 3, 243-254 (1955)

11. Grigorchuk, R.: Symmetrical random walks on discrete groups. In: Multicomponent Random Systems. Advanced Probability Related Topics, vol. 6, pp. 285-325. Dekker, New York (1980)

12. Hirsch, M., Thurston, W.: Foliated bundles, invariant measures and flat manifolds. Ann. Math. 101, 369-390 (1975)

13. Jaerisch, J.: Group-extended Markov systems, amenability, and the Perron-Frobenius operator. Proc. Am. Math. Soc. 143, 289-300 (2015)

14. Kesten, H.: Full Banach mean values on countable groups. Math. Scand. 7, 146-156 (1959)

15. Kitchens, B.: Symbolic Dynamics: One-sided. Two-sided and Countable State Markov Shifts. Springer, Berlin (1998)

16. Manning, A.: Axiom A diffeomorphisms have rational zeta functions. Bull. Lond. Math. Soc. 3, 215220 (1971)

17. Northshield, S.: Cogrowth of regular graphs. Proc. Am. Math. Soc. 116, 203-205 (1992)

18. Northshield, S.: Cogrowth of arbitrary graphs. In: Random Walks and Geometry. de Gruyter, Berlin (2004)

19. Ortner, R., Woess, W.: Non-backtracking random walks and co-growth of graphs. Can. J. Math. 59, 828-844 (2007)

20. Parry, W., Pollicott, M.: Zeta functions and the periodic orbit structure of hyperbolic dynamics. Astérisque 187-188, 1-268 (1990)

21. Paulin, F., Pollicott, M., Schapira, B.: Equilibrium states in negative curvature. Astérisque 373, Soc. Math. France (2015)

22. Perry, P.: Asymptotics of the length spectrum for hyperbolic manifolds of infinite volume. Geom. Funct. Anal. 11, 132-141 (2001)

23. Pollicott, M.: A symbolic proof of a theorem of Margulis on geodesic arcs on negatively curved manifolds. Am. J. Math. 117, 289-305 (1995) 
24. Pollicott, M., Sharp, R.: Orbit counting for some discrete groups acting on simply connected manifolds with negative curvature. Invent. Math. 117, 275-302 (1994)

25. Rees, M.: Checking ergodicity for some geodesic flows with infinite Gibbs measure. Ergod. Theory Dyn. Syst. 1, 107-133 (1981)

26. Roblin, T.: Un théorème de Fatou pour les densités conformes avec applications aux revêtements galoisiens en courbure négative. Israel J. Math. 147, 333-357 (2005)

27. Roblin, T., Tapie, S.: Exposants critiques et moyennabilité. In: Dal'Bo-Milonet, F. (ed. ) Géométrie Ergodique, Monographie No. 43 de l'Enseignement Mathématique, Genève (2013)

28. Sarig, O.: Thermodynamic formalism for countable Markov shifts. Ergod. Theory Dyn. Syst. 19, 1565-1595 (1999)

29. Sarig, O.: Lecture notes on thermodynamic formalism for topological Markov shifts. http://www. wisdom.weizmann.ac.il/ sarigo/TDFnotes. Accessed 3 Nov 2014

30. Stadlbauer, M.: An extension of Kesten's criterion for amenability to topological Markov chains. Adv. Math. 235, 450-468 (2013)

31. Woess, W.: Cogrowth of groups and simple random walks. Arch. Math. (Basel) 41, 363-370 (1983) 